\title{
Domestic Load Characterization through Smart Meter Advance Stratification
}

\author{
Bruce Stephen, Member IEEE, and Stuart Galloway
}

\begin{abstract}
The heterogeneity of domestic loads presents Distribution Network Operators with operational uncertainties which may become problematic as generation capacity shrinks and network infrastructure ages. High resolution meter advances recorded by increasingly ubiquitous Smart Meters can be seen as representing base loads along with aggregations of multiple domestic appliances - in this letter, a Bayesian formulation of the finite mixture paradigm is employed to capture generalizations of these, from which compact representations of domestic load profiles can be formed.
\end{abstract}

Index Terms-- Load modeling, Power Systems, Clustering methods.

\section{DOMESTIC LOAD MONITORING}

$\mathrm{M}$ ANY energy regulatory bodies in the developed world have proposed legislation for the replacement of legacy electricity metering with an Automated Meter Reading (AMR) infrastructure employing Smart Meters. While a key attraction is the alleviation of the need to access the property to bill customers, the detailed domestic load data holds value to both energy retailers, from the perspective of understanding their customers, and distribution network operators (DNOs) from the perspective of understanding and managing the performance of the supply infrastructure to inform optimal network operation. Through regulator OFGEM, the UK government intends a roll out of Smart Meters to all UK domestic properties by 2020 [1]. Smart Meter hardware [1] typically comprises a digital replacement to the induction disk type meter, storing readings at a far higher time resolution and transmits these advances of the cumulated energy use (possibly wirelessly) back to the energy retailer for billing purposes. Trials of Smart Meters in domestic properties have been motivated by a move away from flat rate tariffs to policies such as Critical Peak Pricing (CPP) and Time of Use (TOU) [2] which vary the price paid per kWh dependent on the time of day or the wholesale energy price in order to control domestic loads. In implementing Smart Grid technology, access to more detailed load measurements at additional points on the distribution and low voltage networks gives network operators the ability to establish the extent to which demand must be managed and network infrastructure

Dr. B. Stephen is a Senior Research Fellow in the Advanced Electrical Systems Research Group, Institute of Energy and Environment, University of Strathclyde, Glasgow, G1 1XW (phone: +44 (0)141 548 5864, e-mail: bstephen@eee.strath.ac.uk) must be invested in. Although load profiles are available for planning of network capacity, these can be difficult to keep up to date as consumer lifestyles change and become more energy intensive [3]. Figure 1 shows the diversity of domestic loads captured by Smart Meters in a major UK city over a single fortnight for a number of different properties.

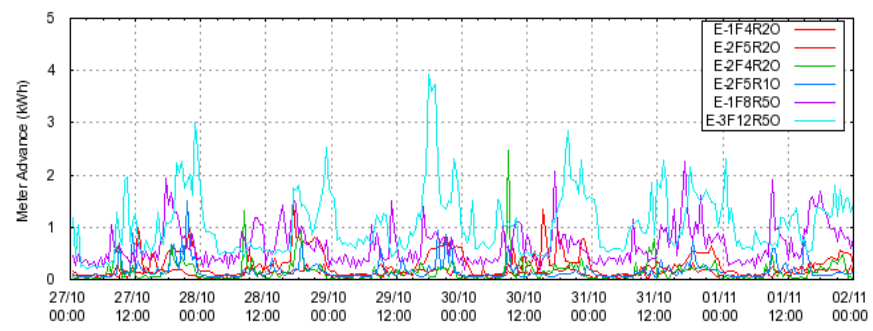

Fig. 1. Half hourly advances from 6 houses of different size and occupancy.

The purpose of this letter is to demonstrate a method of reducing household behavior to a sequence or collection of higher level predicates that enable comparison or inference of data of relevance to the system operator or retailer.

\section{ABSTRACTION OF DOMESTIC LOADS}

Smart Meter data typically captures the aggregated domestic loads accumulated over a 30 minute period. While this offers a previously unknown degree of insight into the behavior in an individual dwelling as an aggregation of appliance loads [4], this data alone cannot offer a representation that allows ready comparison either with other dwellings in the same time period or with itself over a number of different time periods. Figure 1 implies that there are several general advance levels or stratifications present in domestic load data which can be the base load for one household and near the peak for another although 'peak' and 'base' labels at this level of detail are less meaningful. All the DNO should be concerned with is to identify a general reoccurrence of a particular load level. A binning approach would allow this to be achieved but the question of the width and position of these bins remains, making a simple rule based approach problematic. An alternative would be to construct a piecewise model of the probability density, which is essentially a smoothed histogram, and use the pieces to delineate energy usage into a discrete number of strata.

An arbitrary probability density of variable $x$ can be approximated by a linear combination of parametric distributions, known as a finite mixture model. Although it is possible to learn the parameters of these models using maximum likelihood estimation, the pathologies of this 
coupled with the benefits of Bayesian modeling [5] make iterative approximation of a Bayesian model more attractive.

\section{VARIATIONAL BAyesian MiXTURE MODEL}

The general formulation of the posterior distribution of a mixture model with parameters $\Theta, N$ samples of observed data $X$ (such as the meter advances) and unobserved variables $S$ (for example, the energy stratum labels) is as follows:

$$
P(\Theta, S \mid X) \propto P(X \mid \Theta, S) P(\Theta) \prod_{i=1}^{N} P\left(s_{i} \mid \bar{\pi}\right) P(\bar{\pi} \mid \alpha) P(\alpha)
$$

$\pi$ is the vector of mixing proportions which scales each component to best fit the implied distribution and $\alpha$ is the hyperparameter of a Dirichlet prior distribution over the mixing proportions. Although equation (1) is computationally intractable, in [6] it was demonstrated how a Bayesian formulation of a mixture of Gaussian distributions could be learned iteratively from data using Variational Inference. Applying these statistical models to customer meter reads will enable classification of domestic load profiles without the requirement for prior knowledge or rules pertaining to customer behavior.

\section{CAPTURING ENERGY BAND OCCURRENCES}

Using a fortnight of mid-autumn data taken from 6 heterogeneous UK suburban dwellings to provide an example of modern domestic loads, an optimally parameterized mixture model was learned. Using the normalized Free Energy, a measure of model fit proposed in [6], out of a set of models with up to 25 strata tested, the number of stratum was found to be 7 as figure 2 illustrates.

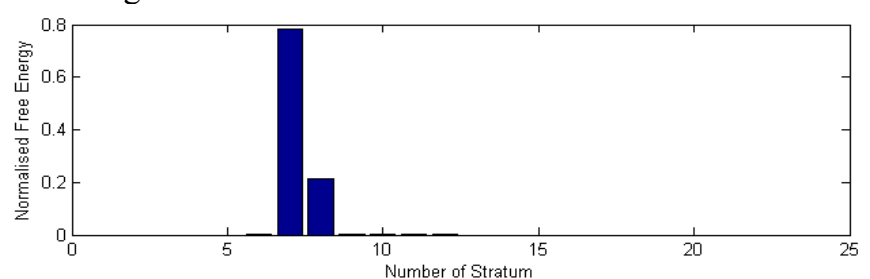

Fig. 2. Identifying the number of strata using normalized Free Energy.

The model Free Energy approach was identified by [6] to provide a less ambiguous indication of the cardinality of mixture models. Horizontal lines on figure 3 show the position of the 7 strata, each represented by a Gaussian mean, with respect to the actual meter advances observed in the data used to learn the model. After they are obtained the strata are ordered low to high according to their associated mean value.

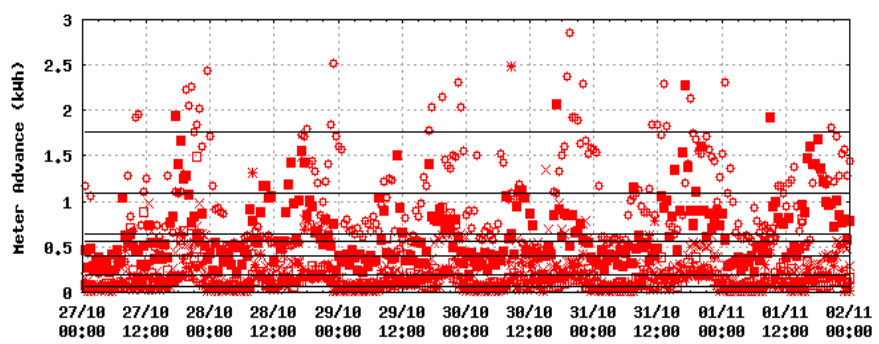

Fig. 3. Exemplar advance data with positions of energy strata superimposed.

Figure 4 shows how the strata counts are inferred daily for a single dwelling through the learned model - it yields the mixture component most likely to have generated each 30 minute advance. Advances from the single property are considered from late summer to early winter, resulting in a set of counts for each stratum showing how much energy in each band the property uses and which bands are out with the observed usage of the dwelling.

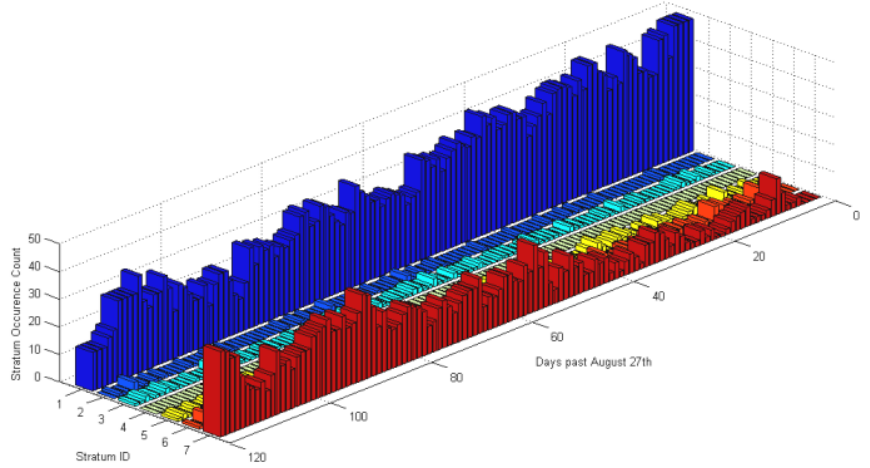

Fig. 4. Time series of energy stratum occurrence counts for a single property. As the season progresses towards winter, the daily stratum population shift - the base load stratum (label 1) occurrences are gradually supplanted by progressively higher peaks (label 7). In the same way, the advances for every property in a given neighborhood could be summed over to give the anticipated count of strata at the neighborhood feeder at a given time period providing the DNO with demand knowledge.

\section{CONCLUSIONS AND FURTHER WORK}

This letter has demonstrated the application of an energy usage stratification technique that can be used as the basis for dynamically learning and recognizing domestic load profiles. By reducing half hourly meter advances from dwellings into occurrence counts of energy use stratum 'labels' allows, in the simplest application, for base and/or peak usage to be tracked. This can be expanded through more sophisticated modeling techniques to models of compositional and time varying behavior of domestic loads. More generally profiling of domestic properties is an important activity in network capacity planning, asset management of network infrastructure, scheduling of generation and tariff design for which the stratification technique has a role.

\section{REFERENCES}

[1] Darby, S. "Smart metering: what potential for householder engagement?", Building Research \& Information, Vol. 38, No. 5, pp. 442 - 457, Routledge 2010.

[2] Mohsenian-Rad, A.H. \& Leon-Garcia, A., "Optimal Residential Load Control With Price Prediction in Real-Time Electricity Pricing Environments", IEEE Transactions on Smart Grid, Vol.1, No.2, pp.120133, September 2010.

[3] Loveday, D.L., Bhamra, T., Tang, T., Haines, V.J.A., Holmes, M.J. \& Green R.J. The energy and monetary implications of the '24/7' 'always on' society. Energy Policy 36, pp. 4639-4645, Elsevier 2008.

[4] Liang, J. Ng, S.K.K., Kendall, G. \& Cheng, J.W.M. Load Signature Study - Part I: Basic Concept, Structure, and Methodology. IEEE Transactions on Power Delivery, Vol. 25, No. 2, April 2010.

[5] D. J. C. MacKay. Probable networks and plausible predictions - a review of practical Bayesian methods for supervised neural networks. Network: Computation in Neural Systems, 6(3), pp.469-505, 1995.

[6] W.D. Penny, S.J. Roberts, Variational Bayes for 1-dimensional mixture models, Technical Report PARG-2000-01, Department of Engineering Science, Oxford University, 2000. 